\title{
On the Boundary between Nonlinear Jump Phenomenon and Linear Response of Hypoid Gear Dynamics
}

\author{
Jun Wang ${ }^{1}$ and Teik C. Lim² \\ ${ }^{1}$ Advanced Components Systems Division, Caterpillar Inc., Peoria, IL 61630, USA \\ ${ }^{2}$ Mechanical Engineering Program, School of Dynamic Systems, University of Cincinnati, 598 Rhodes Hall, \\ P.O. Box 210072, Cincinnati, OH 45221, USA
}

Correspondence should be addressed to Teik C. Lim, teik.lim@uc.edu

Received 27 October 2010; Revised 10 March 2011; Accepted 17 March 2011

Academic Editor: Mohammad Tawfik

Copyright ( $\odot 2011$ J. Wang and T. C. Lim. This is an open access article distributed under the Creative Commons Attribution License, which permits unrestricted use, distribution, and reproduction in any medium, provided the original work is properly cited.

A nonlinear time-varying (NLTV) dynamic model of a hypoid gear pair system with time-dependent mesh point, line-of-action vector, mesh stiffness, mesh damping, and backlash nonlinearity is formulated to analyze the transitional phase between nonlinear jump phenomenon and linear response. It is found that the classical jump discontinuity will occur if the dynamic mesh force exceeds the mean value of tooth mesh force. On the other hand, the propensity for the gear response to jump disappears when the dynamic mesh force is lower than the mean mesh force. Furthermore, the dynamic analysis is able to distinguish the specific tooth impact types from analyzing the behaviors of the dynamic mesh force. The proposed theory is general and also applicable to high-speed spur, helical and spiral bevel gears even though those types of gears are not the primary focus of this paper.

\section{Introduction}

Gear dynamics have been studied intensively as evident from the discussions in [1-5]. In one of the earlier studies, Comparin and Singh investigated the nonlinear frequency response of an impact pair and located the transition conditions for no-impact, single-sided impact, and double-sided impact cases [1]. Later, Kahraman, and Singh examined the nonlinear dynamics of a spur gear pair [2] as well as a geared rotor-bearing system [3] and also studied the interaction between mesh stiffness and clearance nonlinearities [4]. More recently, Cheng and Lim studied the vibratory response of a hypoid geared rotor system with nonlinear time-varying mesh characteristics [5]. From those studies, it is now well known that nonlinear phenomena like jump discontinuity frequently occurs for lightly loaded gear pairs. As the drive load is progressively increased, the response becomes more linear until the jump response completely disappears. Understanding the conditions ahead of time when the geared system will exhibit nonlinear behaviors is highly desirable since the nonlinear dynamic analysis is typically very time consuming. However, to the best of our knowledge this transitional phase has never been studied extensively in the past or fully understood. Hence, the goal of this study is to seek out the conditions dictating the transitional boundary between nonlinear and linear responses. In other word, we would like to know precisely when jump phenomenon stops in advance as drive load goes from light to heavy.

To assist in the above-mentioned analysis, a nonlinear time-varying dynamic model with time-dependent mesh position, line-of-action mesh vectors, mesh stiffness, mesh damping, and backlash nonlinearity is proposed similar to the one employed by the authors in earlier studies $[6,7]$. Based on the model, different levels of torques and damping ratios are applied so that the transition condition between linear and nonlinear responses can be located. The analysis clearly shows that when the dynamic mesh force is greater than mean mesh force, the jump phenomena will occur. On the other hand, if the dynamic mesh force is smaller in value than the mean mesh force, the jump behavior tends to disappear making the response appearing to be quite linear.

\section{Mathematical Model}

The two degrees-of-freedom (DOF) lumped parameter torsional vibration model of a hypoid gear pair as shown 


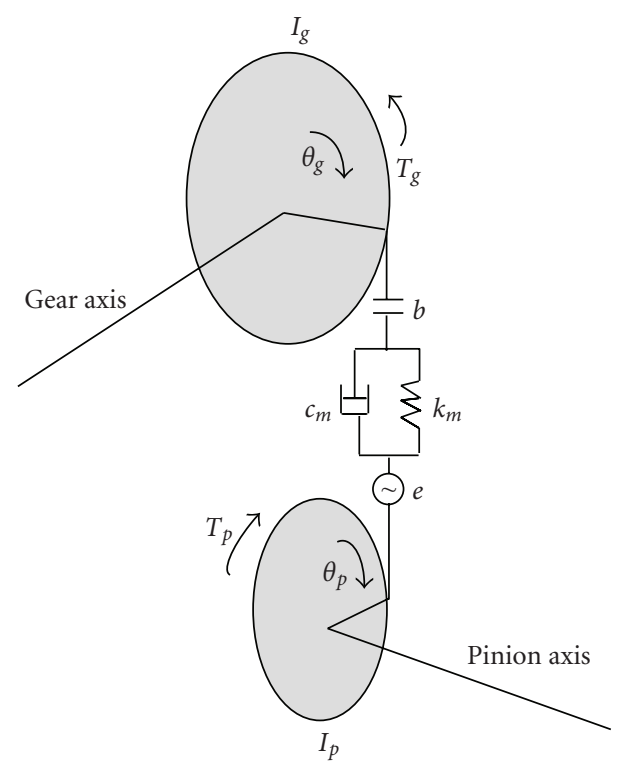

(a)

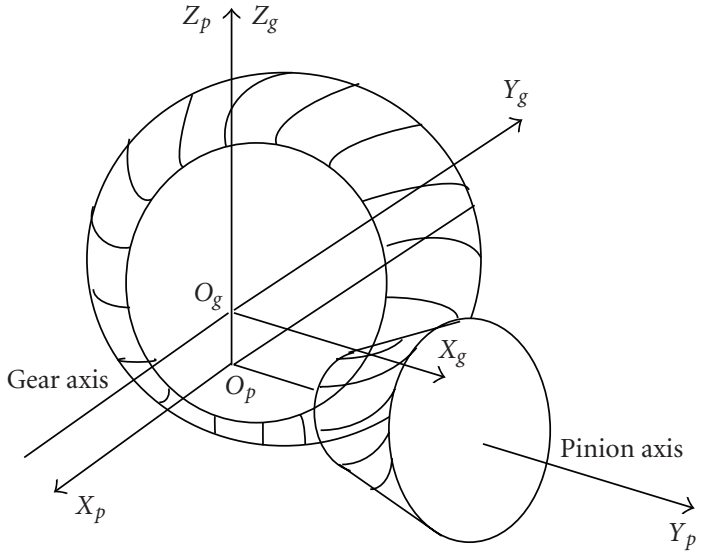

(b)

FIgure 1: (a) Dynamic model of a hypoid gear pair, and (b) pinion and gear coordinate systems.

in Figure 1(a), which was also applied in earlier studies by the authors $[6,7]$, is again assumed for the current study. This simplified representation is chosen over more complex system to allow for the study to focus on the behavior of the boundary between nonlinear jump phenomenon and linear dynamic response. The pinion and gear bodies each are allowed to rotate only. The mesh coupling is represented by a pair of mesh damping and stiffness elements. Using either the Newton's or Lagrangian's methods, the equations of motion can be easily derived as

$$
\begin{gathered}
I_{p} \ddot{\theta}_{p}+\lambda_{p} c_{m} g(\dot{\delta}-\dot{e})+\lambda_{p} k_{m} f(\delta-e)=T_{p}, \\
I_{g} \ddot{\theta}_{g}-\lambda_{g} c_{m} g(\dot{\delta}-\dot{e})-\lambda_{g} k_{m} f(\delta-e)=-T_{g}, \\
f(\delta-e)= \begin{cases}(\delta-e-b), & \delta-e \geq b, \\
0, & -b<\delta-e<b, \\
(\delta-e+b), & \delta-e \leq-b,\end{cases} \\
g(\dot{\delta}-\dot{e})= \begin{cases}(\dot{\delta}-\dot{e}), & \delta-e \geq b, \\
0, & -b<\delta-e<b, \\
(\dot{\delta}-\dot{e}), & \delta-e \leq-b,\end{cases}
\end{gathered}
$$

where $I_{p}$ and $I_{g}$ are the mass moments of inertias of pinion and gear, $T_{p}$ and $T_{g}$ are torque loads a on the pinion and gear, $k_{m}$ and $c_{m}$ are mesh stiffness and mesh damping coefficients, $e$ is unloaded kinematic transmission error, and $2 b$ is the gear backlash. The dynamic transmission error can be written as $\delta=\lambda_{p} \theta_{p}-\lambda_{g} \theta_{g}$, while the directional rotation radius can be expressed as $\lambda_{l}=\vec{n}_{l} \cdot\left(\vec{r}_{l} \times \vec{j}_{l}\right)(l=p, g$ for pinion and gear resp.). Also, $\vec{n}_{l}$ is the unit vector of the line of action along the mesh force direction in the coordinate system $S_{l}$ as shown in Figure $1(\mathrm{~b}), \vec{r}_{l}$ is the position vector of mesh point, and $\vec{j}_{l}$ is the unit vector along the rotating axes of pinion and gear.

By assuming $p=\delta-e,(1 \mathrm{a})$ and (1b) can be combined and simplified into the following form:

$$
\begin{gathered}
m_{e} \ddot{p}+c_{m} g(\dot{p})+k_{m} f(p)=m_{e}\left(\frac{\lambda_{p} T_{p}}{I_{p}}+\frac{\lambda_{g} T_{g}}{I_{g}}-\ddot{e}\right), \\
f(p)= \begin{cases}p-b, & p \geq b, \\
0, & -b<p<b, \\
p+b, & p \leq-b,\end{cases} \\
g(\dot{p})= \begin{cases}0, & -b<p<b, \\
\dot{p}, & \text { else },\end{cases}
\end{gathered}
$$

where $m_{e}=1 /\left(\lambda_{p}^{2} / I_{p}+\lambda_{g}^{2} / I_{g}\right)$. Since mesh damping is generally time varying, proportional mesh damping is assumed here, which is more realistic than simply applying constant damping. Thus, the damping model can be expressed as

$$
\begin{gathered}
\zeta=\frac{c_{m}}{2 \sqrt{k_{m} m_{e m}}}=\frac{q k_{m}}{2 m_{e m} \omega_{n}}=\frac{q \omega_{n}}{2}, \\
c_{m}=q k_{m}=\frac{2 \zeta}{\omega_{n}} k_{m},
\end{gathered}
$$

where $\omega_{n}=\sqrt{k_{m m} / m_{e m}}, m_{e m}=1 /\left(\lambda_{p m}^{2} / I_{p}+\lambda_{g m}^{2} / I_{g}\right)$. 


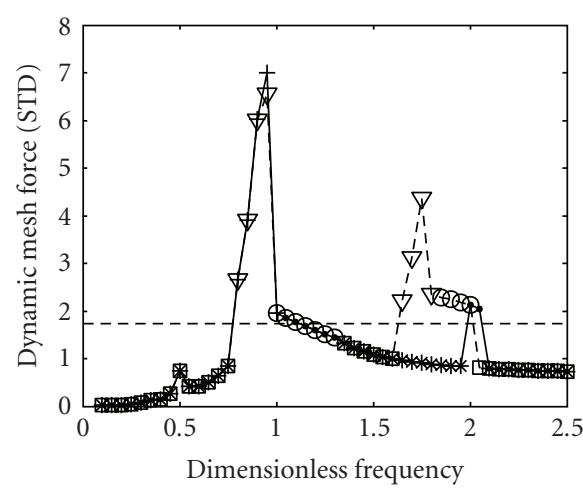

(a)

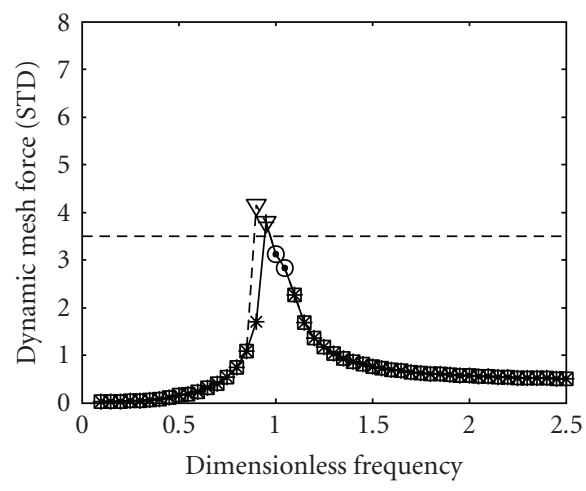

(c)

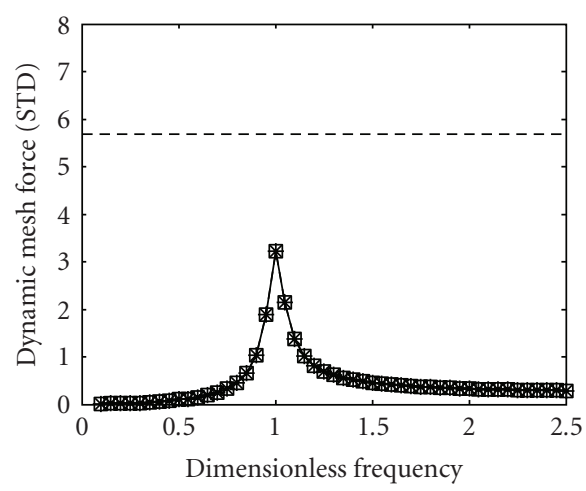

(e)

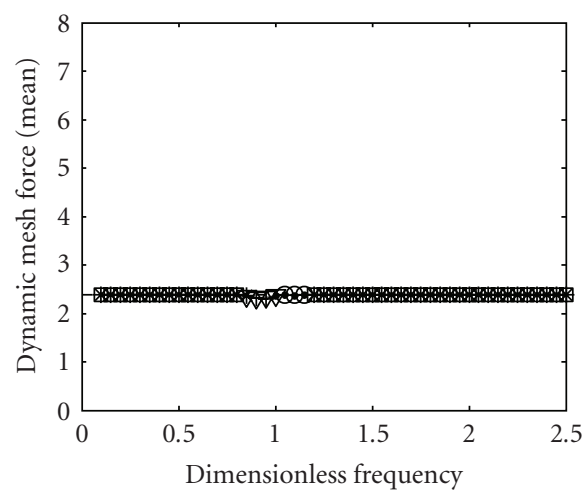

(g)

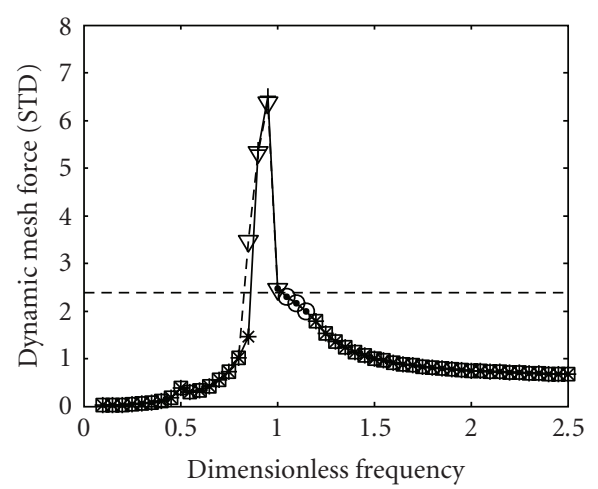

(b)

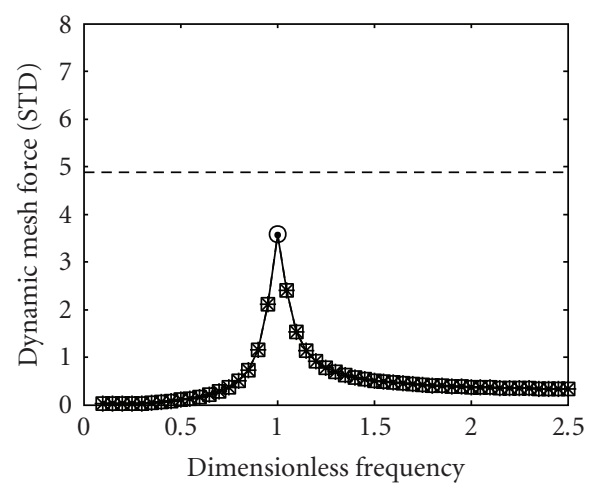

(d)

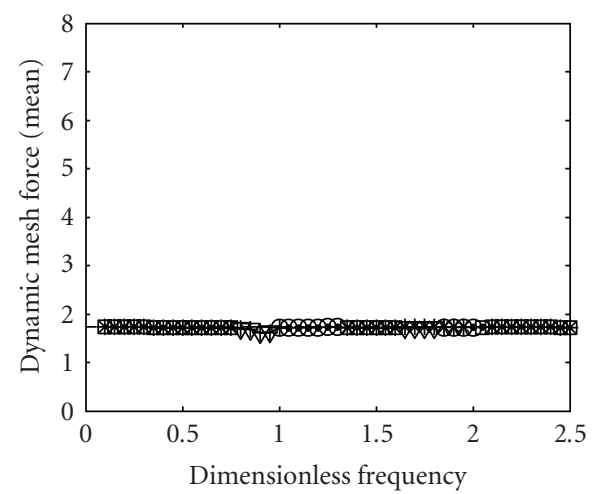

(f)

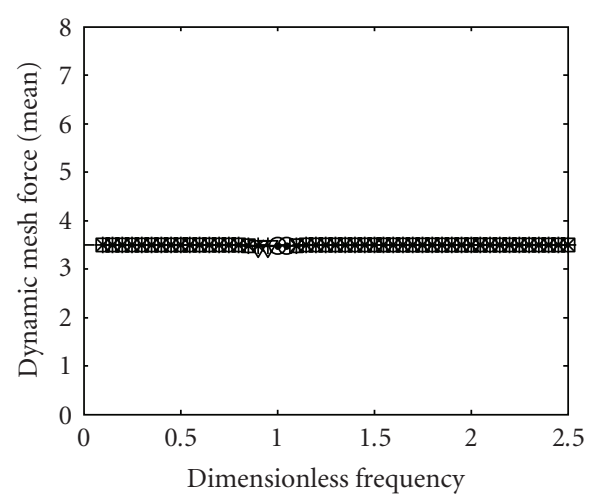

(h)

Figure 2: Continued. 


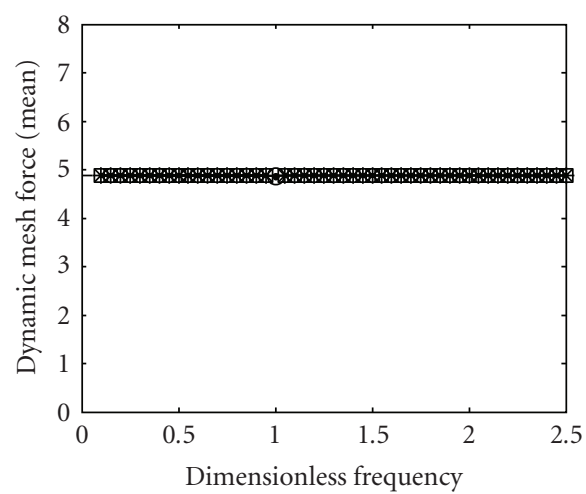

(i)

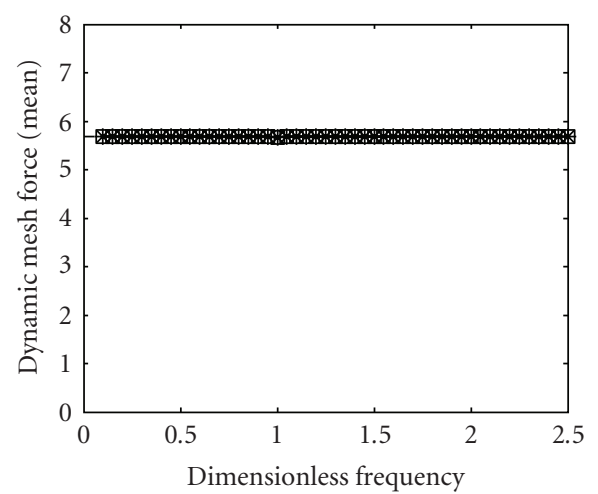

(j)

Figure 2: Dynamic mesh force response for different input torque loads. Dynamic mesh force (STD) is shown in (a) $\widetilde{T}_{p}=0.99$, (b) $\widetilde{T}_{p}=1.37$, (c) $\widetilde{T}_{p}=2.01$, (d) $\widetilde{T}_{p}=2.81$, and (e) $\widetilde{T}_{p}=3.27$, while mean value is given by (f) $\widetilde{T}_{p}=0.99$, (g) $\widetilde{T}_{p}=1.37$, (h) $\widetilde{T}_{p}=2.01$, (i) $\widetilde{T}_{p}=2.81$, and (j) $\widetilde{T}_{p}=3.27 . *: \square$ no impact; $\bullet: \bigcirc$ single-sided impact; $+: \nabla$ double-sided impact; — $~$ : dynamic mesh force (increasing frequency); ...: dynamic mesh force (decreasing frequency); - - -: theoretical static mesh force.

The time-varying mesh parameters in $(2 \mathrm{a}),(2 \mathrm{~b})$, and $(2 \mathrm{c})$ can be described as a superposition of a mean value and a sinusoidal component as shown below:

$$
\begin{gathered}
\lambda_{p}=\lambda_{p m}+\lambda_{p a} \cos \left(\omega t+\phi_{p}\right), \\
\lambda_{g}=\lambda_{g m}+\lambda_{g a} \cos \left(\omega t+\phi_{g}\right), \\
k_{m}=k_{m m}+k_{m a} \cos \left(\omega t+\phi_{k}\right), \\
e=e_{a} \cos \left(\omega t+\phi_{e}\right) .
\end{gathered}
$$

The dynamic mesh force can be computed from

$$
F_{d}=c_{m} g(\dot{p})+k_{m} f(p)=2 \zeta \frac{k_{m}}{\omega_{n}} g(\dot{p})+k_{m} f(p) .
$$

From the torque and directional rotation radii, the mean value of the mesh force can be calculated from

$$
F_{s}=\frac{T_{p}}{\lambda_{p m}}=\frac{T_{g}}{\lambda_{g m}} .
$$

By further assuming dimensionless parameters $\tilde{p}=p / b$, $\tilde{t}=\omega_{n} t, \tilde{\omega}=\omega / \omega_{n}, \tilde{\lambda}_{p}=\lambda_{p} / \lambda_{p m}, \tilde{\lambda}_{g}=\lambda_{g} / \lambda_{g m}, \tilde{k}=k_{m} / k_{m m}$, and $\tilde{e}=e / b$, the dimensionless form of (2a) can be obtained as

$$
\begin{gathered}
\tilde{p}^{\prime \prime}+2 \zeta \frac{\tilde{\lambda}_{p}^{2}+\eta \tilde{\lambda}_{g}^{2}}{1+\eta} \widetilde{k}_{g}\left(\tilde{p}^{\prime}\right)+\frac{\tilde{\lambda}_{p}^{2}+\eta \tilde{\lambda}_{g}^{2}}{1+\eta} \tilde{k} f(\tilde{p}) \\
=\widetilde{T}_{p} \tilde{\lambda}_{p}+\widetilde{T}_{g} \tilde{\lambda}_{g}-\tilde{e}^{\prime \prime}, \\
f(\tilde{p})= \begin{cases}\tilde{p}-1, & \tilde{p} \geq 1, \\
0, & -1<\tilde{p}<1, \\
\tilde{p}+1, & \tilde{p} \leq-1,\end{cases} \\
g\left(\tilde{p}^{\prime}\right)= \begin{cases}0, & -1<\tilde{p}<1, \\
\tilde{p}^{\prime}, & \text { else, }\end{cases}
\end{gathered}
$$

where $\eta=\lambda_{g m}^{2} I_{p} / \lambda_{p m}^{2} I_{g}, \widetilde{T}_{p}=\lambda_{p m} T_{p} / b \omega_{n}^{2} I_{p}$, and $\widetilde{T}_{g}=\eta \widetilde{T}_{p}$. The dimensionless mesh parameters are described as

$$
\begin{gathered}
\tilde{\lambda}_{p}=1+\tilde{\lambda}_{p a} \cos \left(\tilde{\omega} \tilde{t}+\phi_{p}\right), \\
\tilde{\lambda}_{g}=1+\tilde{\lambda}_{g a} \cos \left(\tilde{\omega} \tilde{t}+\phi_{g}\right), \\
\tilde{k}=1+\tilde{k}_{a} \cos \left(\tilde{\omega} \tilde{t}+\phi_{k}\right), \\
\tilde{e}=\tilde{e}_{a} \cos \left(\tilde{\omega} \tilde{t}+\phi_{e}\right) .
\end{gathered}
$$

Subsequently the dimensionless dynamic mesh force can be derived as

$$
\widetilde{F}_{d}=2 \zeta \tilde{k} g\left(\tilde{p}^{\prime}\right)+\tilde{k} f(\tilde{p}),
$$

and the dimensionless mean value mesh force can be calculated from

$$
\widetilde{F}_{s}=(1+\eta) \widetilde{T}_{p}
$$

The numerical integration method applying an explicit Runge-Kutta (4) and (5) formulation is employed to compute the response from (8a) since there is no analytical method available. The computed time domain response $\tilde{p}$ is then applied to calculate the dynamic mesh force using (10). From the predicted time history response function, the frequency spectrum can be obtained by taking the standard deviation (STD) and mean values of time series data at each operating frequency. From here onwards, the STD value is considered as the amplitude of dynamic mesh force.

\section{Dynamic Analysis}

Using the proposed dynamic model, a typical rear axle hypoid gear set is analyzed. The basic system parameters of this rear axle unit are given in Table 1, which were 


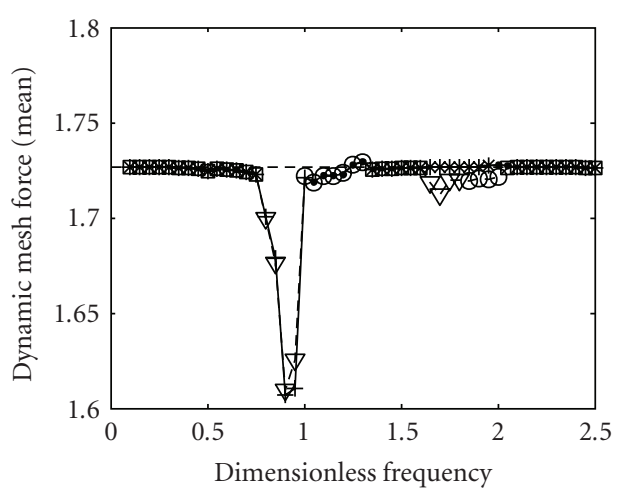

(a)

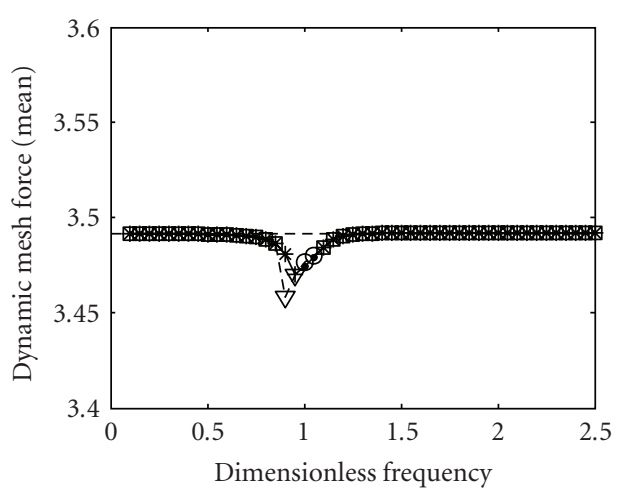

(c)

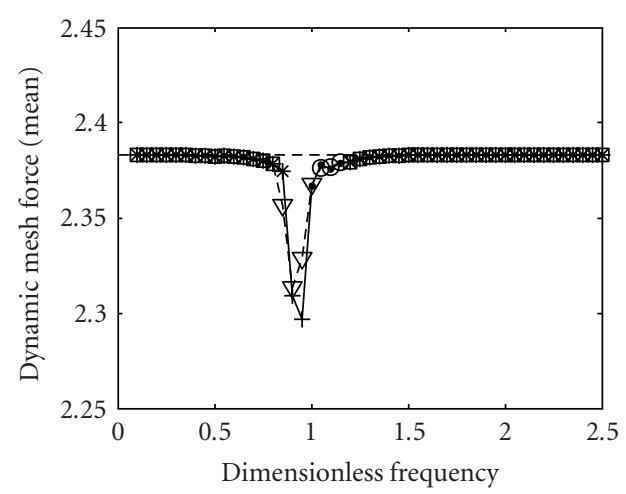

(b)

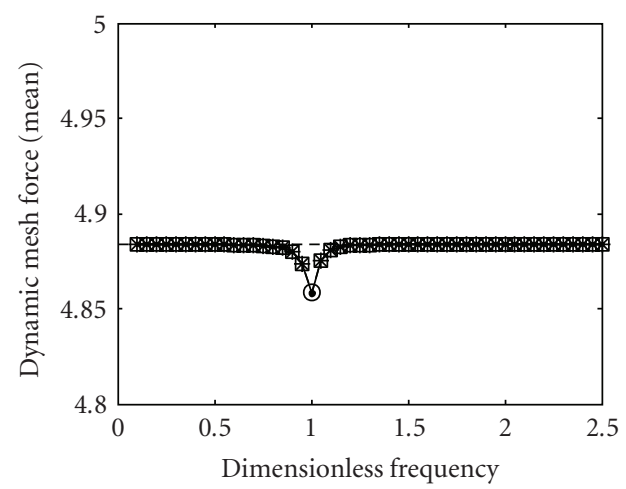

(d)

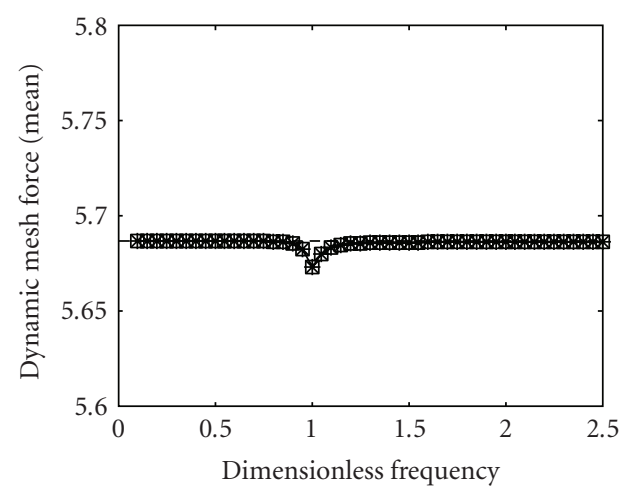

(e)

FIgURE 3: Mean value of mesh force for different input torque loads. (a) $\widetilde{T}_{p}=0.99$, (b) $\widetilde{T}_{p}=1.37$, (c) $\widetilde{T}_{p}=2.01$, (d) $\widetilde{T}_{p}=2.81$, and (e) $\widetilde{T}_{p}=3.27 . *: \square$ no impact; $\bullet: \bigcirc$ single-sided impact; $+: \nabla$ double-sided impact; $—$ : mean value for increasing frequency; ...: mean value for decreasing frequency; - - -: theoretical static mesh force.

also applied in an earlier numerical study by the authors [6]. Tooth contact analysis is first conducted to obtain the necessary mesh parameters for five different loads as shown in Table 2. The procedure for computing mesh parameters obtained from output results of a tooth contact analysis [8] is described in an earlier publication by the authors [9]. The extracted mesh parameters are readily employed in the dynamic model. For brevity, details of the process will not be repeated here.
3.1. Frequency Domain Analysis. The dynamic mesh force response calculated using the above procedure for different mean loads is shown in Figure 2. Here mesh damping ratio of $\zeta=0.04$ is used. As expected, when the torque load increases, the mean value of mesh force increases too. In fact, the mean value of mesh force response is very close to the theoretical static mesh force derived from the input torque load and is almost frequency invariant compared to the dynamic mesh force that varies significantly with 


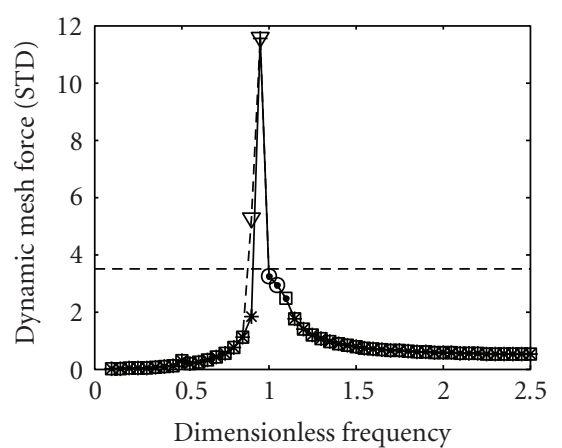

(a)

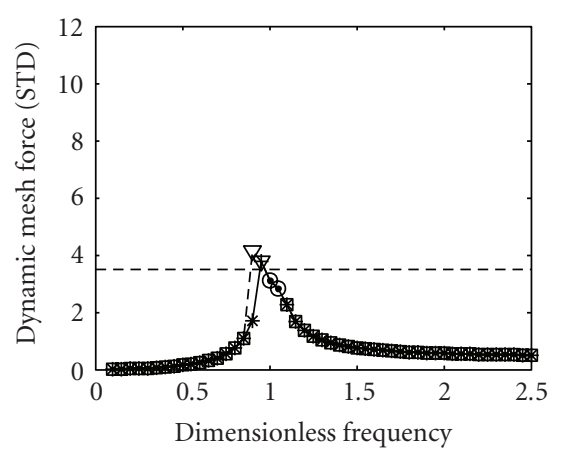

(c)

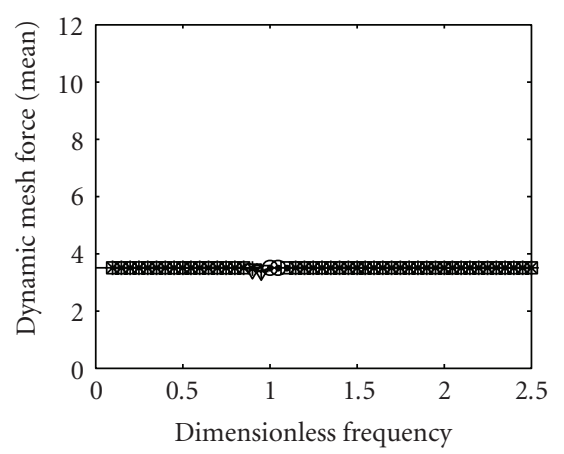

(e)

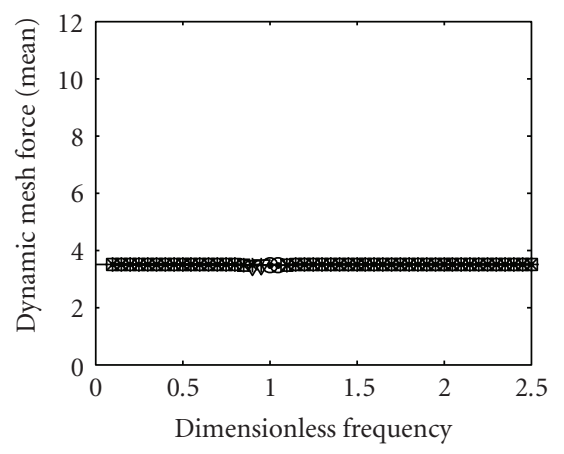

(g)

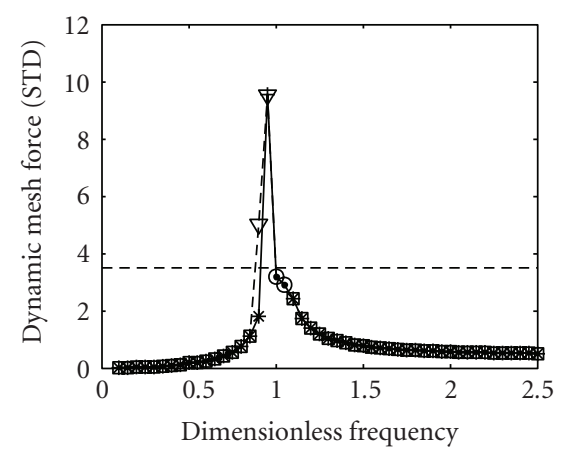

(b)

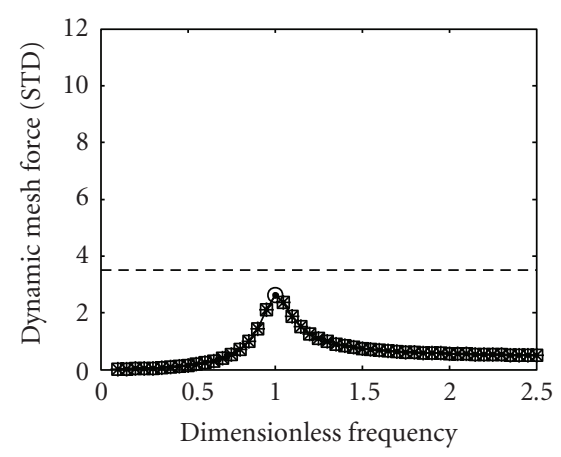

(d)

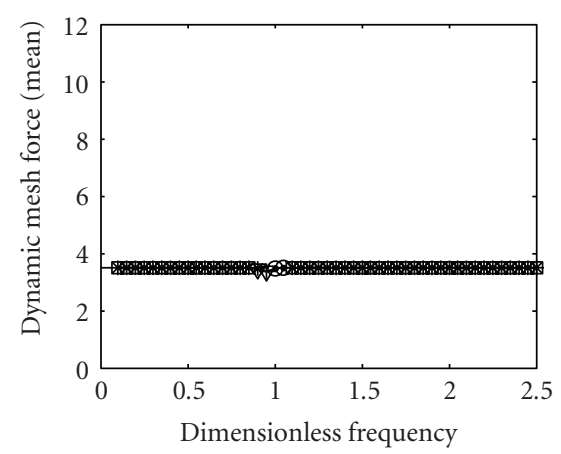

(f)

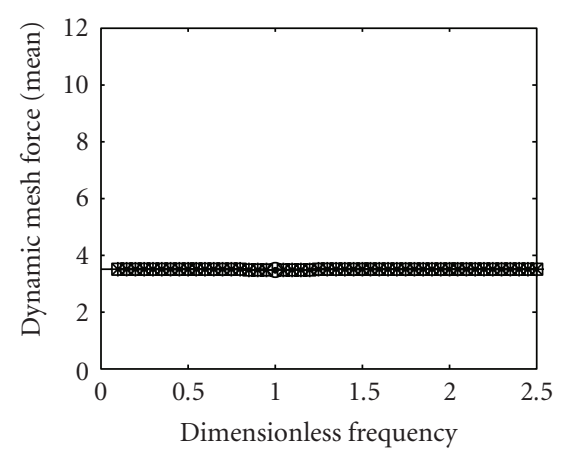

(h)

FIgURE 4: Dynamic mesh force response for different mesh damping ratios. Dynamic mesh force (STD) is shown in (a) $\zeta=0.01$, (b) $\zeta=0.02$, (c) $\zeta=0.04$, and (d) $\zeta=0.08$, while the mean value is given by (e) $\zeta=0.01$, (f) $\zeta=0.02,(\mathrm{~g}) \zeta=0.04$, and (h) $\zeta=0.08$. $*$ : $\square$ no impact; $\bullet: \bigcirc$ single-sided impact; $+: \nabla$ double-sided impact; — _ dynamic mesh force (increasing frequency); ...: dynamic mesh force (decreasing frequency); - - : theoretical static mesh force. 


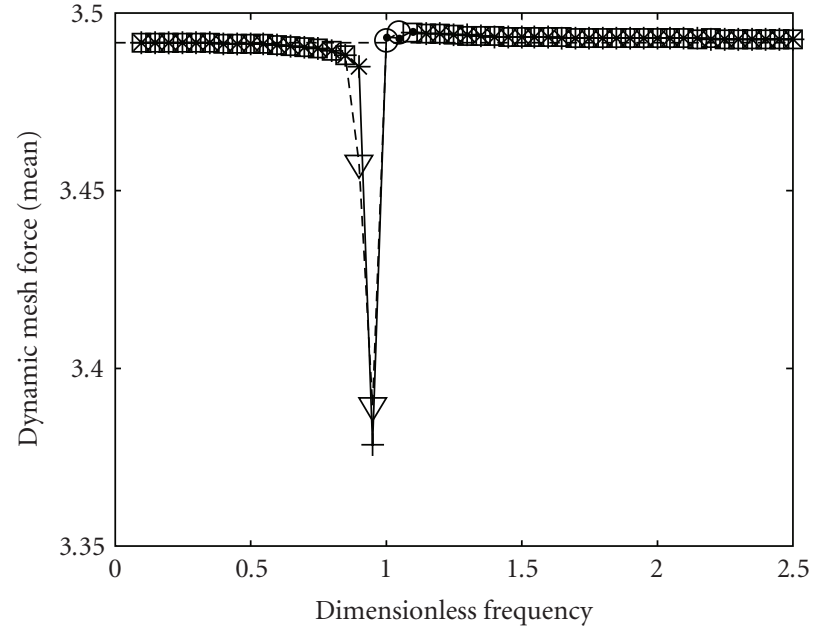

(a)

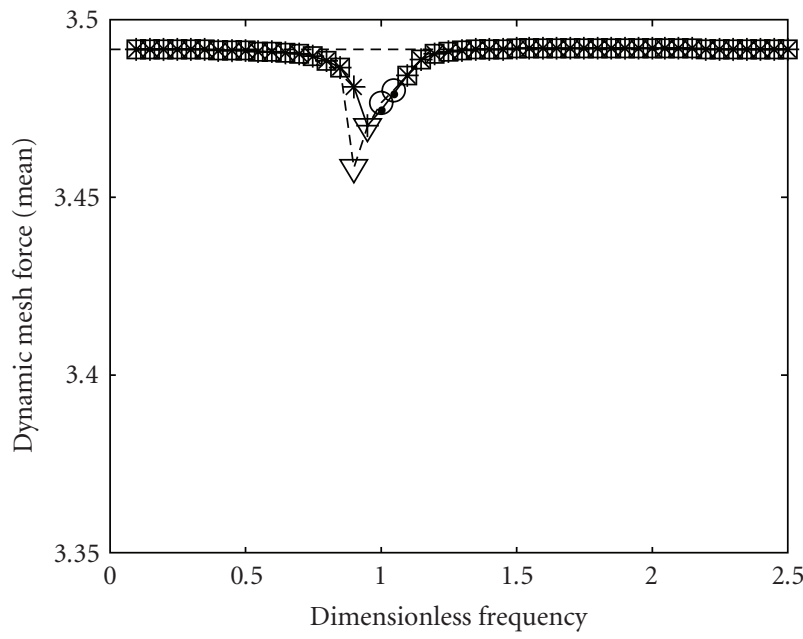

(c)

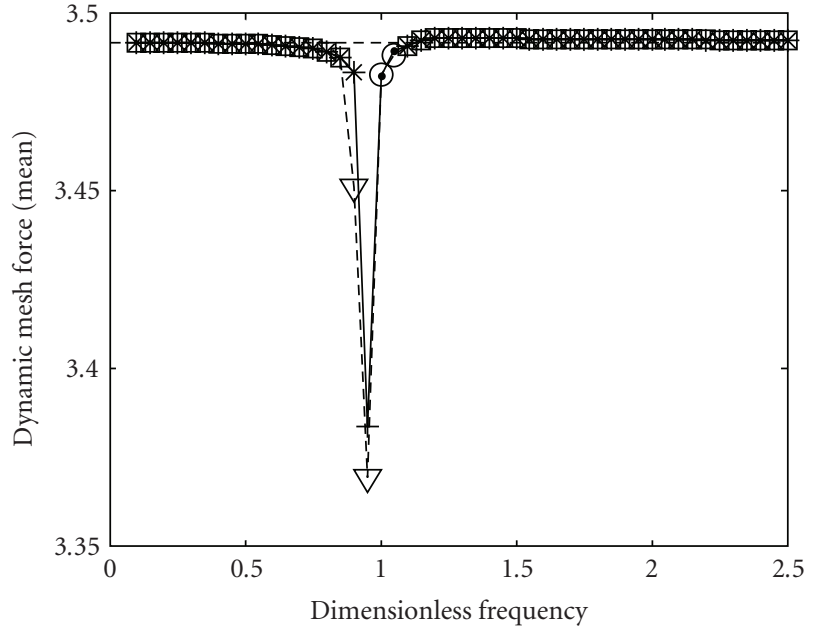

(b)

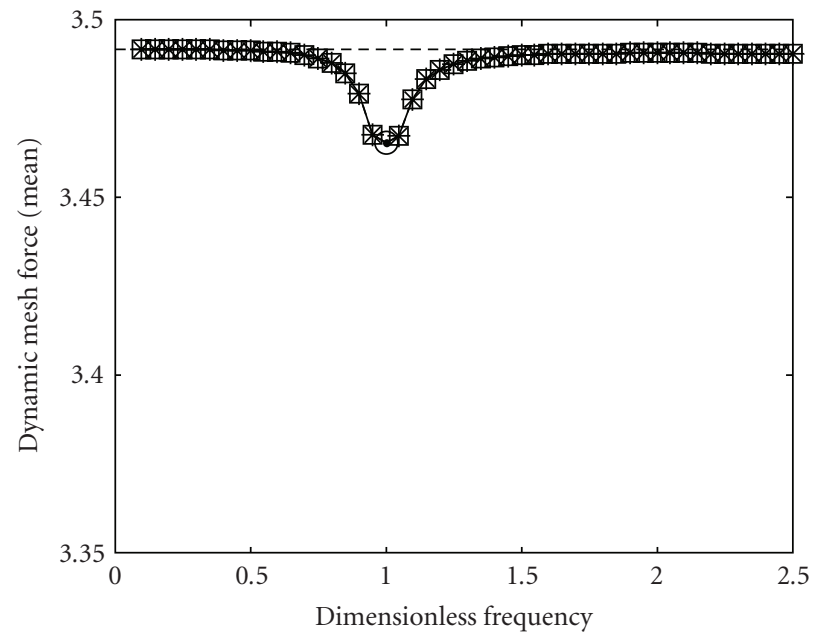

(d)

Figure 5: Mean value of mesh force response for different mesh damping ratios. (a) $\zeta=0.01$, (b) $\zeta=0.02$, (c) $\zeta=0.04$, and (d) $\zeta=0.08$. $*$ : $\square$ no impact; $\bullet: \bigcirc$ single-sided impact; $+: \nabla$ double-sided impact; ——: mean value for increasing frequency; ...: mean value for decreasing frequency; - - -: theoretical static mesh force.

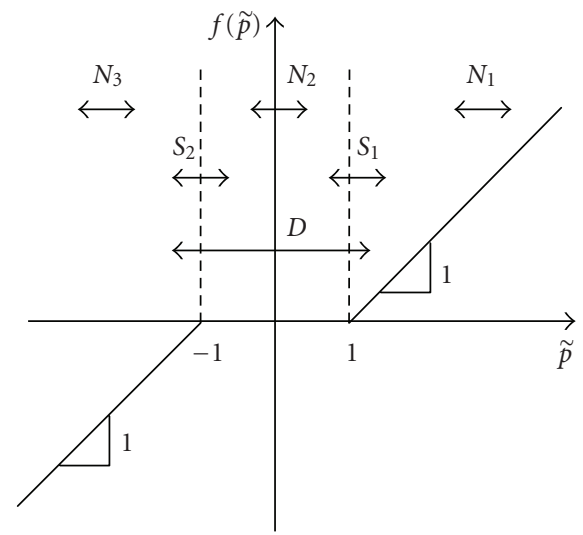

FIGURE 6: Illustration of different tooth impact types. No impact $\left(N_{1}, N_{2}\right.$, and $\left.N_{3}\right)$, single-sided impact $\left(S_{1}, S_{2}\right)$, and double-sided impact $(D)$. 


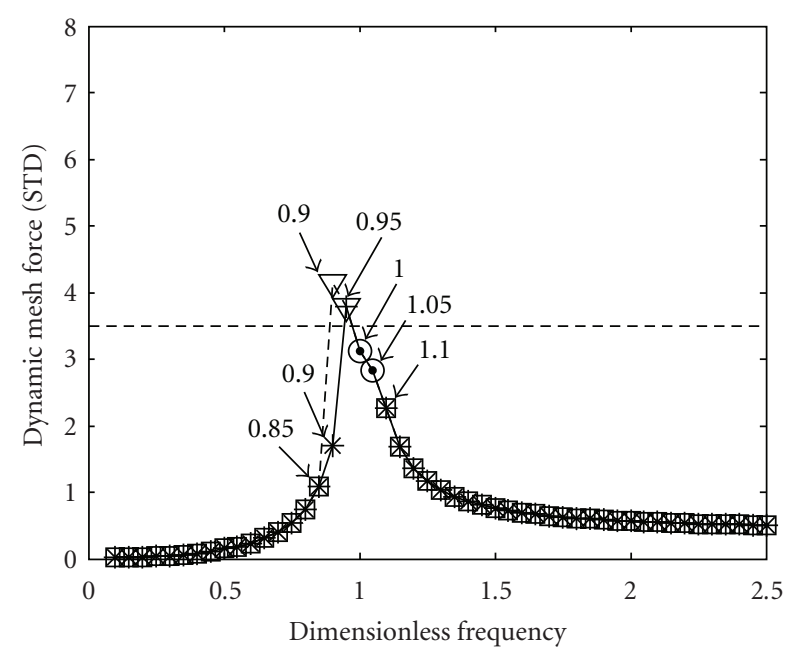

Figure 7: Dynamic mesh force (STD) for $\widetilde{T}_{p}=2.01 . *, \square$ no impact; $\bullet, \bigcirc$ single-sided impact; $+: \nabla$ double-sided impact; - : dynamic mesh force (RMS) for increasing frequency; .... dynamic mesh force (RMS) for decreasing frequency; - - -: theoretical static mesh force.

TABle 1: System parameters for a typical automotive rear axle hypoid gear pair.

\begin{tabular}{lc}
\hline Number of pinion teeth & 10 \\
Number of gear teeth & 43 \\
Pinion offset $(\mathrm{m})$ & 0.0318 \\
Gear pitch radius $(\mathrm{m})$ & 0.168 \\
Pinion pitch radius $(\mathrm{m})$ & 0.048 \\
Mass moment of inertia of pinion $\left(\mathrm{kg}-\mathrm{m}^{2}\right)$ & 0.002 \\
Mass moment of inertia of gear $\left(\mathrm{kg}-\mathrm{m}^{2}\right)$ & 0.05 \\
\hline
\end{tabular}

frequency. Also, the dynamic mesh force appears to decrease with increasing input torque. A more in-depth analysis of the dynamic mesh force results are discussed next.

At $\widetilde{T}_{p}=0.99$, several jump discontinuities and very rich single and double-sided tooth impacts can be observed at the primary resonance and higher frequencies. As $\widetilde{T}_{p}$ is increased to 1.37 , only two jumps are seen in which one is a softening type (lower frequency) and the other is a hardening one (upper frequency). At the same time, less tooth impacts occur around the primary resonance. For the case of $\widetilde{T}_{p}=2.01$ only a softening type jump, and very few single and double-sided tooth impact points appear near the primary resonance. At $\widetilde{T}_{p}=2.81$ there is no jump response observed and only a single-sided tooth impact is found. As $\widetilde{T}_{p}$ is increased further to 3.27 , the dynamic response over the entire frequency range shown is completely void of jump phenomenon and tooth impacts.

From the results, an interesting finding is that when the dynamic mesh force is greater than the mean value of mesh force or the theoretical static mesh force, jump discontinuity can be seen. On the other hand, when the dynamic mesh force is lower than the mean value of mesh force or the
TABLE 2: Dimensionless dynamic parameters of a typical automotive rear axle hypoid gear pair.

\begin{tabular}{lccccc}
\hline Parameter symbols & \multicolumn{5}{c}{ Numerical values } \\
\hline$\tilde{T}_{p}$ & 0.99 & 1.37 & 2.01 & 2.81 & 3.27 \\
$\eta$ & 0.740 & 0.740 & 0.740 & 0.740 & 0.740 \\
$\tilde{\lambda}_{p a 1}$ & 0.015 & 0.014 & 0.010 & 0.006 & 0.004 \\
$\phi_{p 1}$ & $0.25 \pi$ & $0.24 \pi$ & $0.22 \pi$ & $0.15 \pi$ & $0.05 \pi$ \\
$\tilde{\lambda}_{g a 1}$ & 0.013 & 0.013 & 0.009 & 0.005 & 0.004 \\
$\phi_{g 1}$ & $0.25 \pi$ & $0.24 \pi$ & $0.22 \pi$ & $0.15 \pi$ & $0.06 \pi$ \\
$\tilde{k}_{a}$ & 0.231 & 0.137 & 0.053 & 0.0462 & 0.0458 \\
$\phi_{k}$ & $-0.33 \pi$ & $-0.37 \pi$ & $-0.57 \pi$ & $-0.93 \pi$ & $-0.98 \pi$ \\
$\tilde{e}_{a 1}$ & 0.49 & 0.49 & 0.49 & 0.49 & 0.49 \\
$\phi_{e 1}$ & $0.74 \pi$ & $0.74 \pi$ & $0.74 \pi$ & $0.74 \pi$ & $0.74 \pi$ \\
\hline
\end{tabular}

theoretical static mesh force, virtually no jump discontinuity is seen. This is because the mean value of mesh force is able to keep the meshing teeth engaged all the time when its magnitude is higher than the dynamic counterpart that tends to cause the meshing teeth to lose contact. The results of mean value of mesh force response for different levels of input torque load are shown as a closed-up view in Figure 3. Again, as seen in these results, as the drive torque load is increased, the mean mesh force level increases. However, its variation decreases, yielding a more linear characteristic.

The effect of damping is analyzed next. Figure 4 shows the dynamic mesh force response for different mesh damping ratios. From these plots, it can be seen that as $\zeta$ is increased, the dynamic mesh force tends to decrease, while mean value does not change at all. This is consistent with the results depicted in (11). At the same time, occurrence of jump discontinuity and tooth impacts lessen. Also, the mean value of mesh force is very close to the theoretical static mesh force and changes very little. Similarly, the results again show that as dynamic mesh force becomes larger than the mean value of mesh force, jump response appears, while no evidence of jump response is seen when the dynamic mesh force is lower than the mean value.

The effect of mesh damping on the mean value of mesh force is shown in Figure 5. Note that these plots represent a closed-up view of the ones shown in Figure 4. Here, it can be seen that as mesh damping ratio $\zeta$ is increased, the mean value changes very little and its variation tends to decrease. This former result is unlike the previously seen effect of drive torque load, but the latter observation is the same as the trend of drive torque load effect.

3.2. Time Domain Analysis. Due to the design of backlash in the hypoid gear set, there are three classes of tooth meshing cases that are no impact, single-sided impact, and doublesided impact as illustrated in Figure 6. The zone between -1 and 1 is backlash zone. So there are three types of no impact $\left(N_{1}, N_{2}\right.$, and $\left.N_{3}\right)$, two types of single-sided impact $\left(S_{1}\right.$ and $S_{2}$ ), and one type of double-sided impact $(D)$. These tooth 


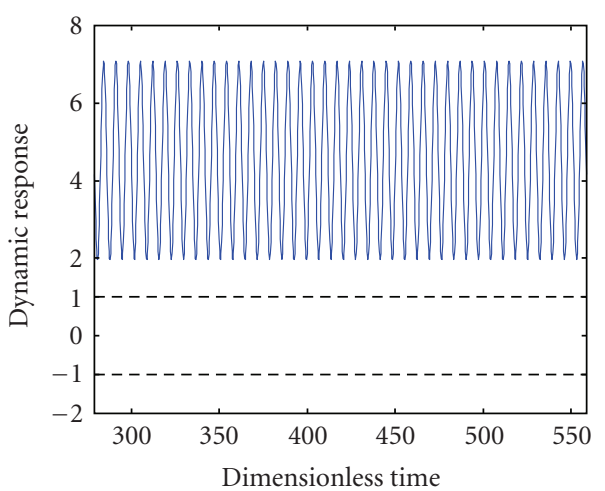

(a)

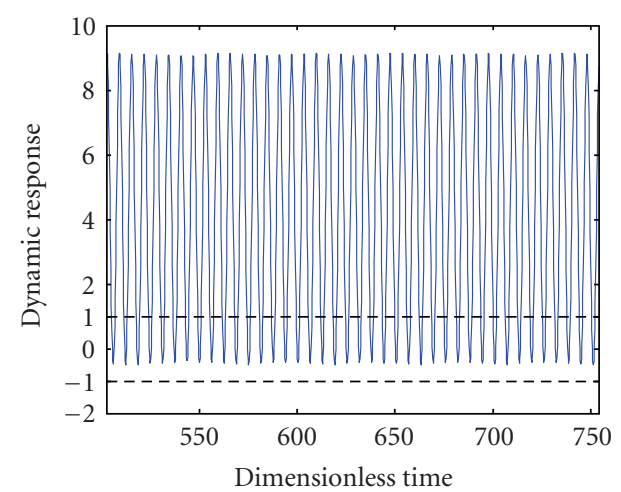

(c)

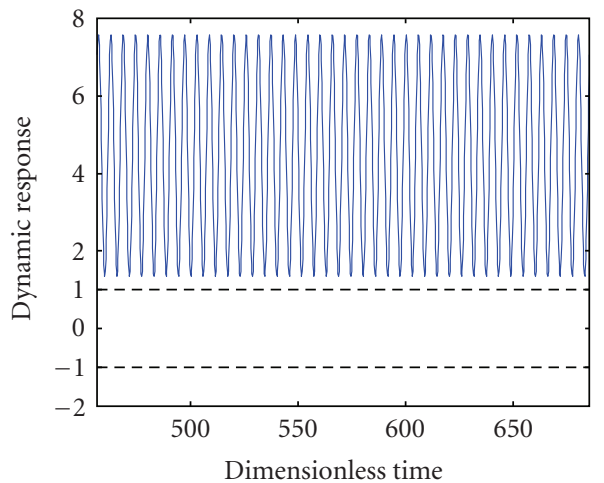

(e)

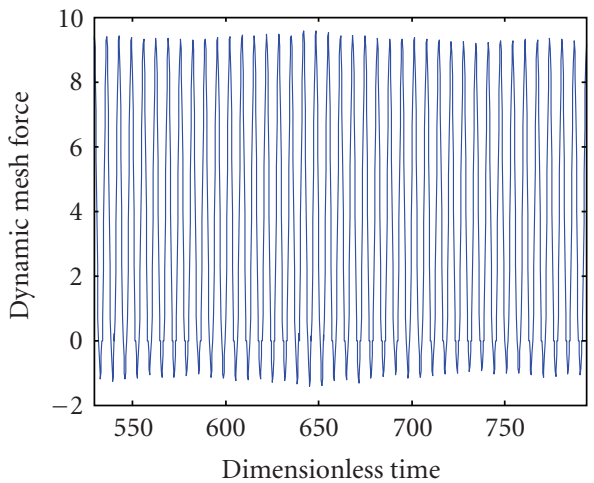

(g)

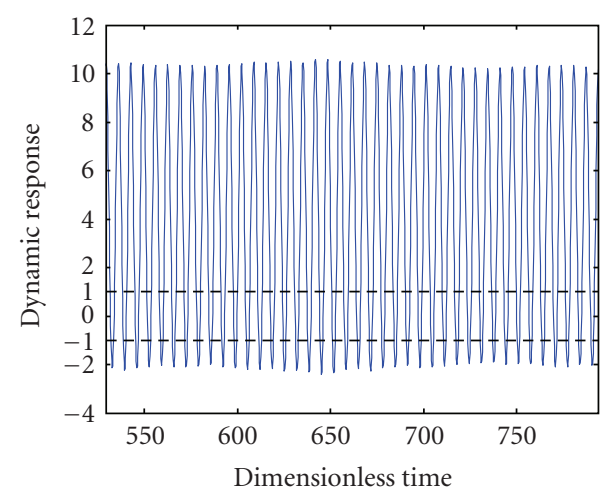

(b)

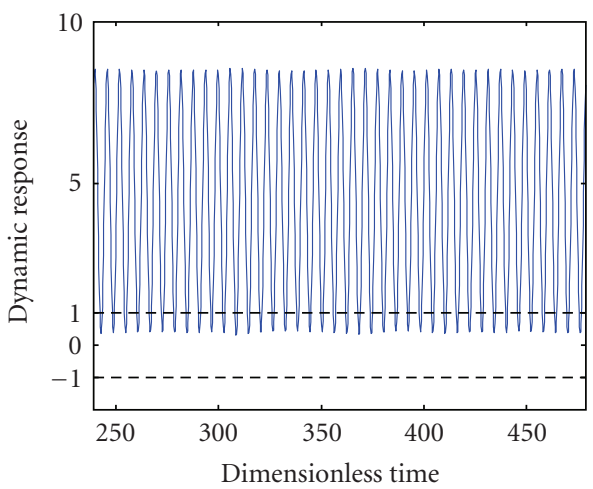

(d)

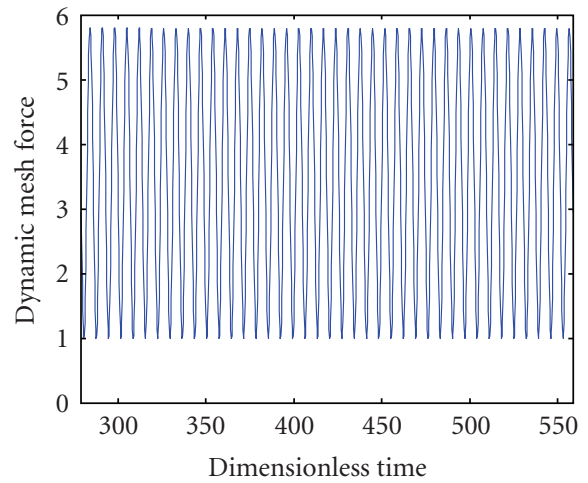

(f)

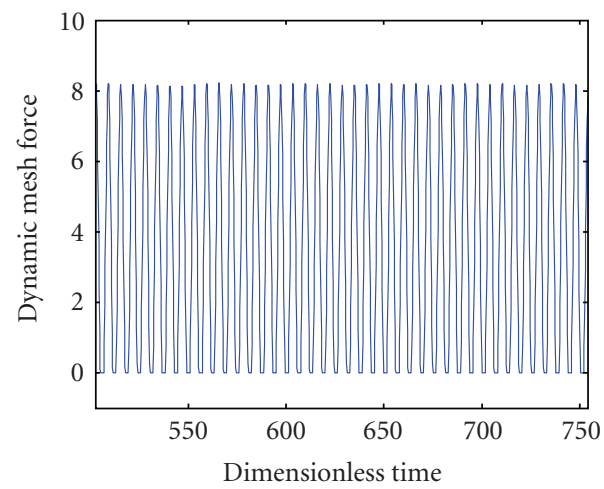

(h)

FIgURE 8: Continued. 


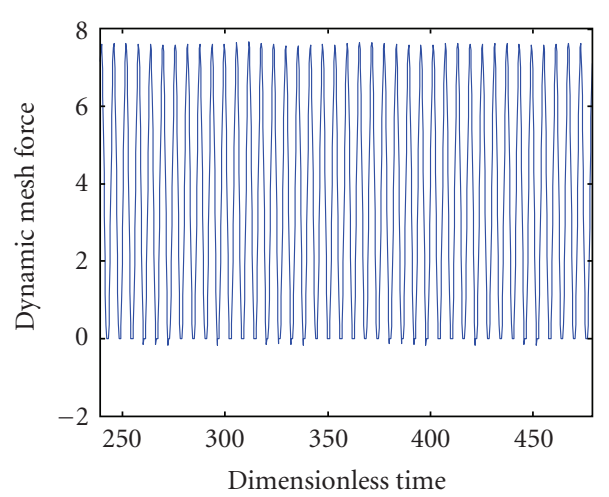

(i)

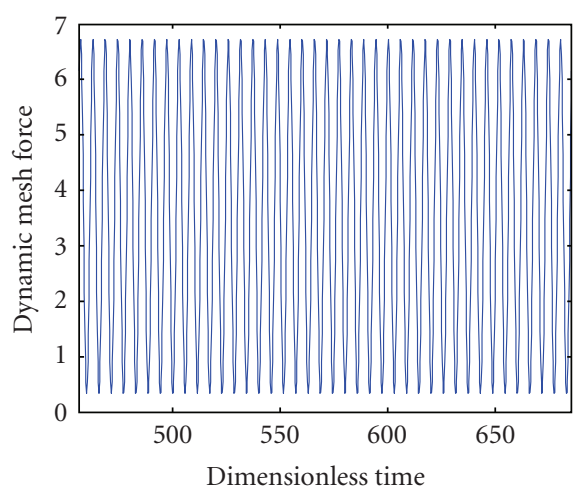

(j)

FIGURE 8: Time history plots of gear response for increasing mesh frequencies. Dynamic response: (a) $\tilde{\omega}=0.9$, (b) $\tilde{\omega}=0.95$, (c) $\tilde{\omega}=1.0$, (d) $\widetilde{\omega}=1.05$, and (e) $\widetilde{\omega}=1.1$. Dynamic mesh force: (f) $\widetilde{\omega}=0.9$, (g) $\widetilde{\omega}=0.95$, (h) $\widetilde{\omega}=1.0$, (i) $\widetilde{\omega}=1.05$, and (j) $\widetilde{\omega}=1.1$.

impact behaviors were first reported by Comparin and Singh in 1989 [1] for parallel axis gears. For nonparallel axis gears, there are no known prior studies. The current study attempts to fill this gap using the hypoid gear design as the example case. In the ensuing discussion, let us consider the predicted dynamic mesh force spectrum for $\widetilde{T}_{p}=2.01$ as shown in Figure 7.

For the case of $\tilde{T}_{p}=2.01$ as shown in Figure 7 , the time history plots of dynamic response for increasing mesh frequencies from 0.9 to 1.1 are shown in a set of plots in Figure 8. In the plot given by Figure 8 (a) for the case of $\widetilde{\omega}=0.9$, the time history response shown is of type $N_{1}$ where no tooth impact response occurs. The corresponding time history plot of the dynamic mesh force at this frequency is shown in Figure $8(\mathrm{f})$. From this figure, it is seen that the trough value of dynamic mesh force is above zero. To explain this observation, consider (10) and (8b) that show the dynamic mesh force being dominated by $\tilde{k} f(\tilde{p})$. Since $f(\tilde{p})$ is greater than zero for $N_{1}$ type no impact response, the dynamic mesh force must therefore be greater than zero. Figures $8(\mathrm{~b})$ and $8(\mathrm{~g})$ for $\widetilde{\omega}=0.95$ shows the time history response of type $D$ double-sided tooth impact behavior. It shows that the trough value of dynamic mesh force reaches below zero because $f(\tilde{p})$ is less than zero for $\tilde{p}<-1$. Figure $8(\mathrm{c})$ shows the time history of $S_{1}$ type single-sided tooth impact response at $\widetilde{\omega}=1.0$, with the corresponding dynamic mesh force shown in Figure $8(\mathrm{~h})$. Here, the trough value of dynamic mesh force is equal to zero because $f(\tilde{p})=$ 0 for $-1<\tilde{p}<1$. This is found to be similar for the time history plots of $S_{1}$ type single-sided tooth impact response and dynamic mesh force at $\widetilde{\omega}=1.05$, as shown in Figures $8(\mathrm{~d})$ and $8(\mathrm{i})$, respectively. At $\widetilde{\omega}=1.1$ as shown in Figures $8(\mathrm{e})$ and $8(\mathrm{j})$, the response goes back to $N_{1}$ type no tooth impact behavior.

Time history plots of dynamic response for decreasing mesh frequencies from 1.1 to 0.85 are shown in Figure 9. In this set of plots, Figure 9 (a) shows time history of $N_{1}$ type no tooth impact response at $\widetilde{\omega}=1.1$ and Figure $9(\mathrm{~g})$ shows the corresponding time history of dynamic mesh force in which the trough value is above zero. As $\widetilde{\omega}$ is decreased to 1.05 , the gear response becomes $S_{1}$ type single-sided tooth impact as depicted by the time history response in Figures 9(b) and 9 (h) in which the trough value is equal to zero. Similar $S_{1}$ type single-sided tooth impact response occurs at $\tilde{\omega}=1.0$ as shown in Figures 9(c) and 9(i). At $\widetilde{\omega}=0.95$ and 0.9 , doublesided tooth impacts occur and the trough value of dynamic mesh force reaches below zero as shown in Figures 9(d), 9(j), $9(\mathrm{e})$, and $9(\mathrm{k})$, respectively. Note that the negative mesh force implies a reversal of its vector along the line of action due to the tooth in question back-colliding with the preceding tooth of the mating gear. As $\widetilde{\omega}$ is further decreased to 0.85 given by Figures 9(f) and 9(1), $N_{1}$ type no tooth impact response appears and the trough value of dynamic mesh force is well above zero.

From the above analysis, it is shown that for $N_{1}$ type no tooth impact case, the trough value of dynamic mesh force is greater than zero; for $S_{1}$ type single-sided tooth impact case, the trough value of dynamic mesh force is equal to zero; and finally for $D$ type double-sided tooth impact case, the trough value of dynamic mesh force reaches below zero. Furthermore, it can be concluded that for $N_{2}$ type no tooth impact case (where no mesh contact at all), the dynamic mesh force will always be equal to zero; for $N_{3}$ type no tooth impact case (where the tooth-tooth engagement happens between the current and preceding tooth of the mating gear), the peak value of dynamic mesh force will be less than zero implying a mesh vector in the opposite direction from the $N_{1}$ case; for $S_{2}$ type single-sided tooth impact case, the peak value of dynamic mesh force will be equal to zero since the current tooth in question never makes contact with the tooth on the mating gear it is designed to engage with.

\section{Concluding Remarks}

A nonlinear time-varying torsional vibration model of a hypoid gear pair system with time-dependent mesh point, line-of-action vector, mesh stiffness, mesh damping, and backlash nonlinearity is formulated to study the condition 


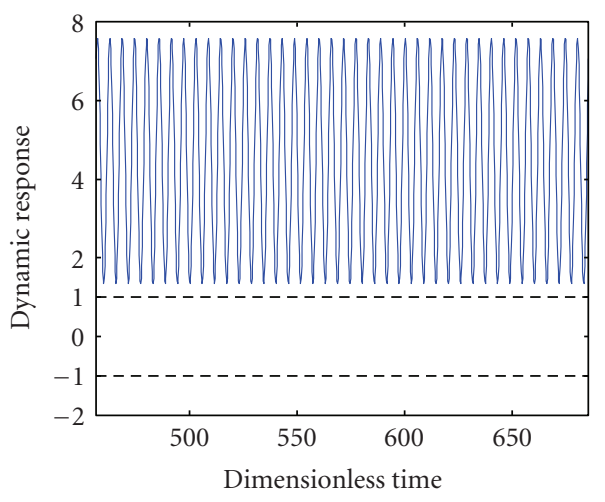

(a)

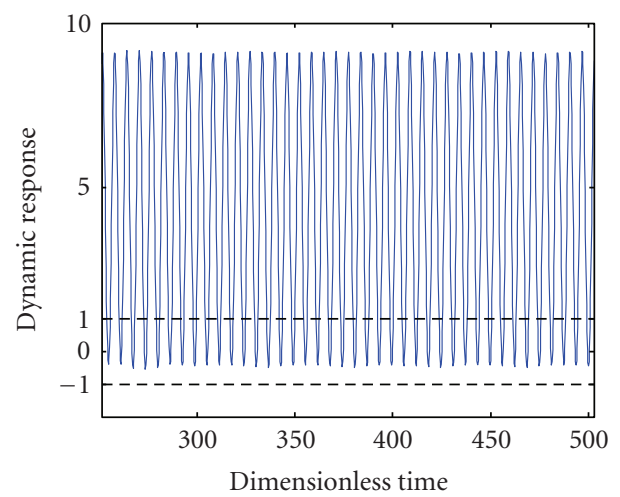

(c)

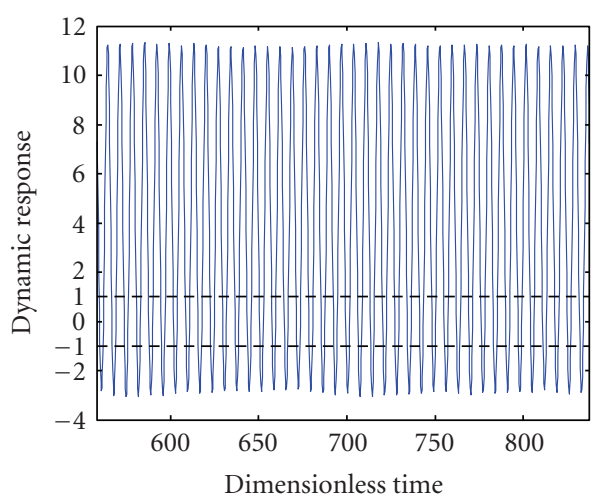

(e)

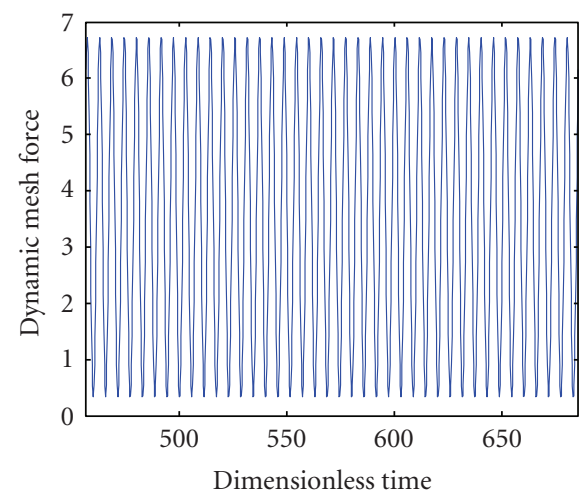

(g)

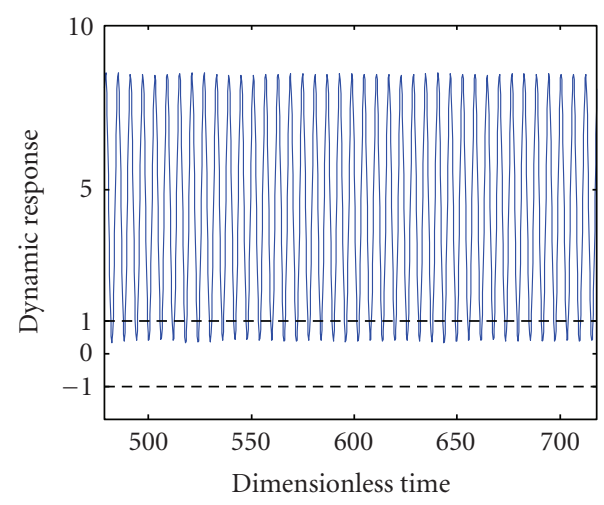

(b)

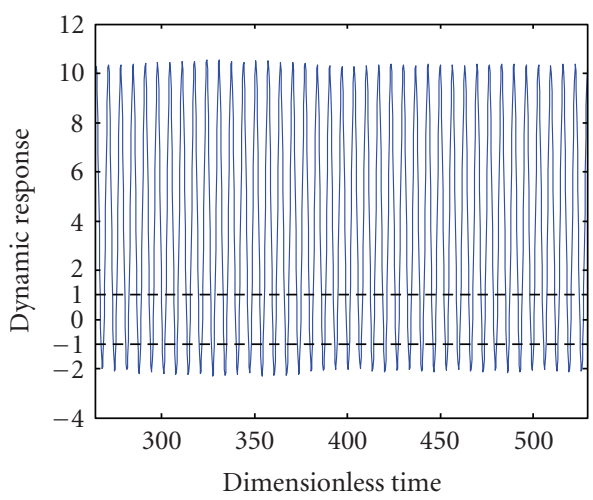

(d)

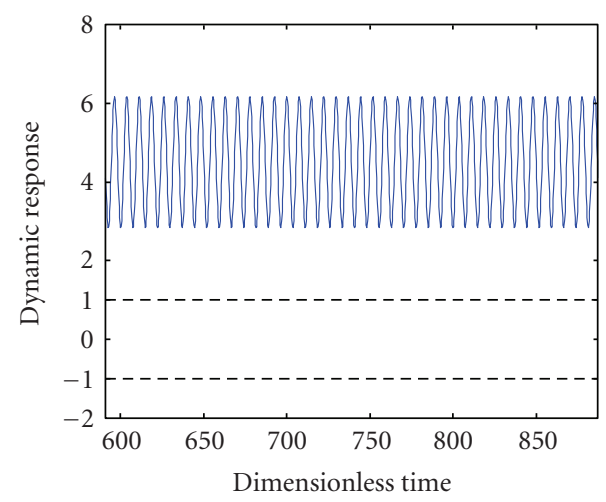

(f)

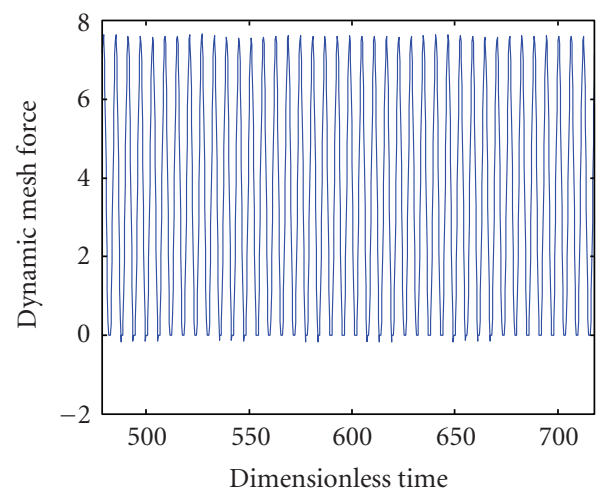

(h)

FIgURE 9: Continued. 


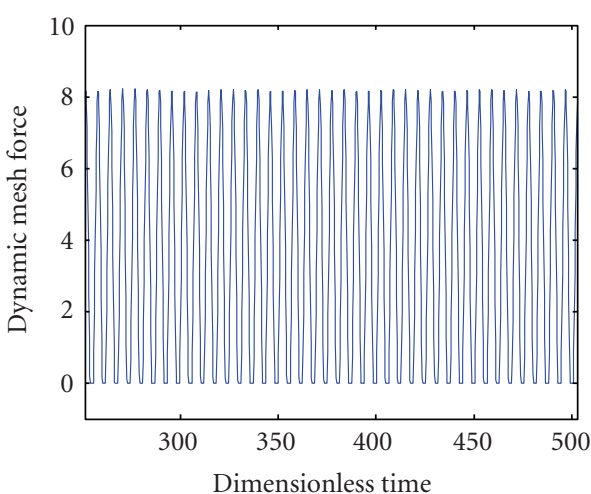

(i)

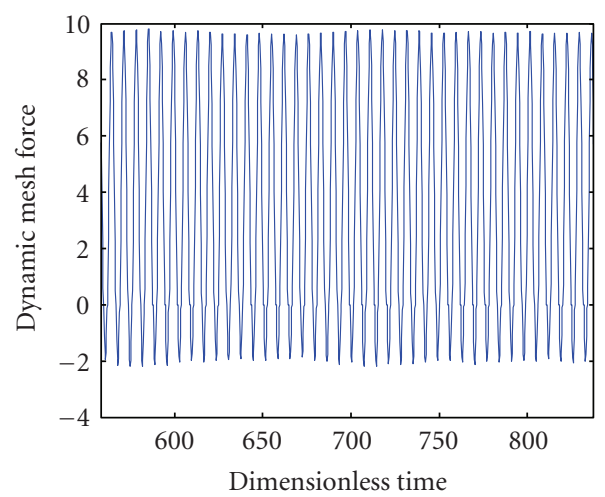

(k)

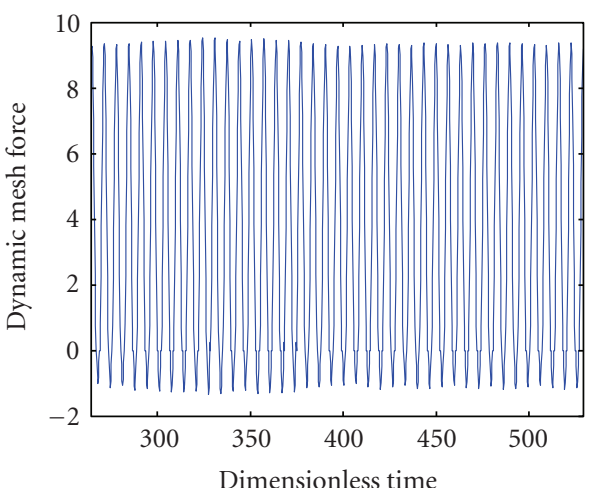

(j)

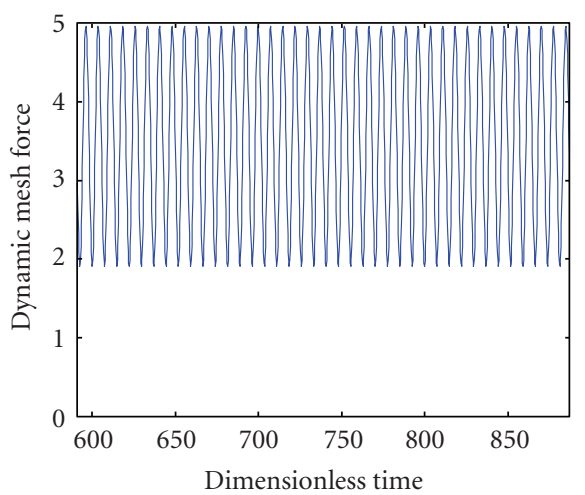

(l)

FIGURE 9: Time history plots of gear response for decreasing mesh frequencies. Dynamic response: (a) $\tilde{\omega}=1.1$, (b) $\widetilde{\omega}=1.05$, (c) $\widetilde{\omega}=1.0$, (d) $\widetilde{\omega}=0.95$, (e) $\tilde{\omega}=0.9$, and (f) $\widetilde{\omega}=0.85$. Dynamic mesh force: (g) $\widetilde{\omega}=1.1$, (h) $\widetilde{\omega}=1.05$, (i) $\widetilde{\omega}=1.0$, (j) $\widetilde{\omega}=0.95$, (k) $\widetilde{\omega}=0.9$, and (l) $\widetilde{\omega}=0.85$.

dictating the boundary between nonlinear jump phenomena and linear responses, that is, when jump discontinuity begins to disappear as mean load is increased from light to heavy levels. An interesting finding is that when the dynamic mesh force exceeds the mean value of mesh force, jump discontinuity will appear, but when the dynamic mesh force is lower than the mean value of mesh force, jump response is less likely to occur.

Based on the time domain analysis results, it is shown that for $N_{1}$ type no tooth impact case, the trough value of dynamic mesh force is greater than zero; for $N_{2}$ type no tooth impact case, the dynamic mesh force is always equal to zero; for $N_{3}$ type no tooth impact case, the peak value of dynamic mesh force is less than zero; for $S_{1}$ type singlesided tooth impact case, the trough value of dynamic mesh force is equal to zero; for $S_{2}$ type single-sided tooth impact case, the peak value of dynamic mesh force is equal to zero; for $D$ type double-sided tooth impact case, the dynamic mesh force reaches both above and below zero. From these observations, it is clear that different tooth impact types can be distinguished by the dynamic mesh force response behavior.

\section{Nomenclature}

$$
\begin{array}{ll}
b: & \text { Gear backlash } \\
c_{m}: & \text { Mesh damping coefficient } \\
e: & \text { Unloaded kinematic transmission error } \\
f: & \text { Nonlinear displacement function } \\
I_{p}, I_{g}: & \text { Mass moments of inertias of pinion and gear } \\
\vec{j}_{l}: & \text { Unit vector along pinion or gear rotating axis } \\
k, k_{m}: & \text { Mesh stiffness } \\
m_{e}: & \text { Equivalent mass } \\
\vec{n}_{l}: & \text { Unit normal vector of mesh point } \\
p: & \text { Difference between dynamic and kinematic } \\
\vec{r}_{l}: & \text { transmission errors } \\
S_{l}: & \text { Position vector of mesh point } \\
t: & \text { Time } \\
T_{p}, T_{g}: & \text { Mean value of pinion and gear torque loads } \\
\delta: & \text { Dynamic transmission error } \\
\lambda_{l}: & \text { Directional rotation radius } \\
\omega: & \text { Excitation frequency }
\end{array}
$$


$\zeta$ : Mesh damping ratio

$\eta$ : Dimensionless torque ratio

$\phi$ : Phase angle.

\section{Subscripts}

$l: \quad$ Label for pinion $(l=p)$ and gear $(l=g)$

$a$ : Fundamental harmonic

$m$ : Mean

$n$ : Natural.

\section{Superscripts}

$\sim:$ Dimensionless quantities

$\rightarrow$ : Vector quantities

': Derivative with respect to time.

\section{References}

[1] R. J. Comparin and R. Singh, "Non-linear frequency response characteristics of an impact pair," Journal of Sound and Vibration, vol. 134, no. 2, pp. 259-290, 1989.

[2] A. Kahraman and R. Singh, "Non-linear dynamics of a spur gear pair," Journal of Sound and Vibration, vol. 142, no. 1, pp. 49-75, 1990.

[3] A. Kahraman and R. Singh, "Non-linear dynamics of a geared rotor-bearing system with multiple clearances," Journal of Sound and Vibration, vol. 144, no. 3, pp. 469-506, 1991.

[4] A. Kahraman and R. Singh, "Interactions between time-varying mesh stiffness and clearance non-linearities in a geared system," Journal of Sound and Vibration, vol. 146, no. 1, pp. 135-156, 1991.

[5] Y. Cheng and T. C. Lim, "Dynamics of hypoid gear transmission with nonlinear time-varying mesh characteristics," Journal of Mechanical Design, Transactions of the ASME, vol. 125, no. 2, pp. 373-382, 2003.

[6] J. Wang, T. C. Lim, and M. Li, "Dynamics of a hypoid gear pair considering the effects of time-varying mesh parameters and backlash nonlinearity," Journal of Sound and Vibration, vol. 308, no. 1-2, pp. 302-329, 2007.

[7] J. Wang and T. C. Lim, "Effect of tooth mesh stiffness asymmetric nonlinearity for drive and coast sides on hypoid gear dynamics," Journal of Sound and Vibration, vol. 319, no. 3-5, pp. 885-903, 2009.

[8] S. Vijayakar, Contact Analysis Program Package: Calyx, Advanced Numerical Solutions, Hilliard, Ohio, 2003.

[9] T. C. Lim and J. Wang, "Effects of assembly errors on hypoid gear mesh and dynamic response," in Proceedings of the ASME Power Transmission and Gearing Conference (DETC '05), Long Beach, Calif, USA, 2005. 

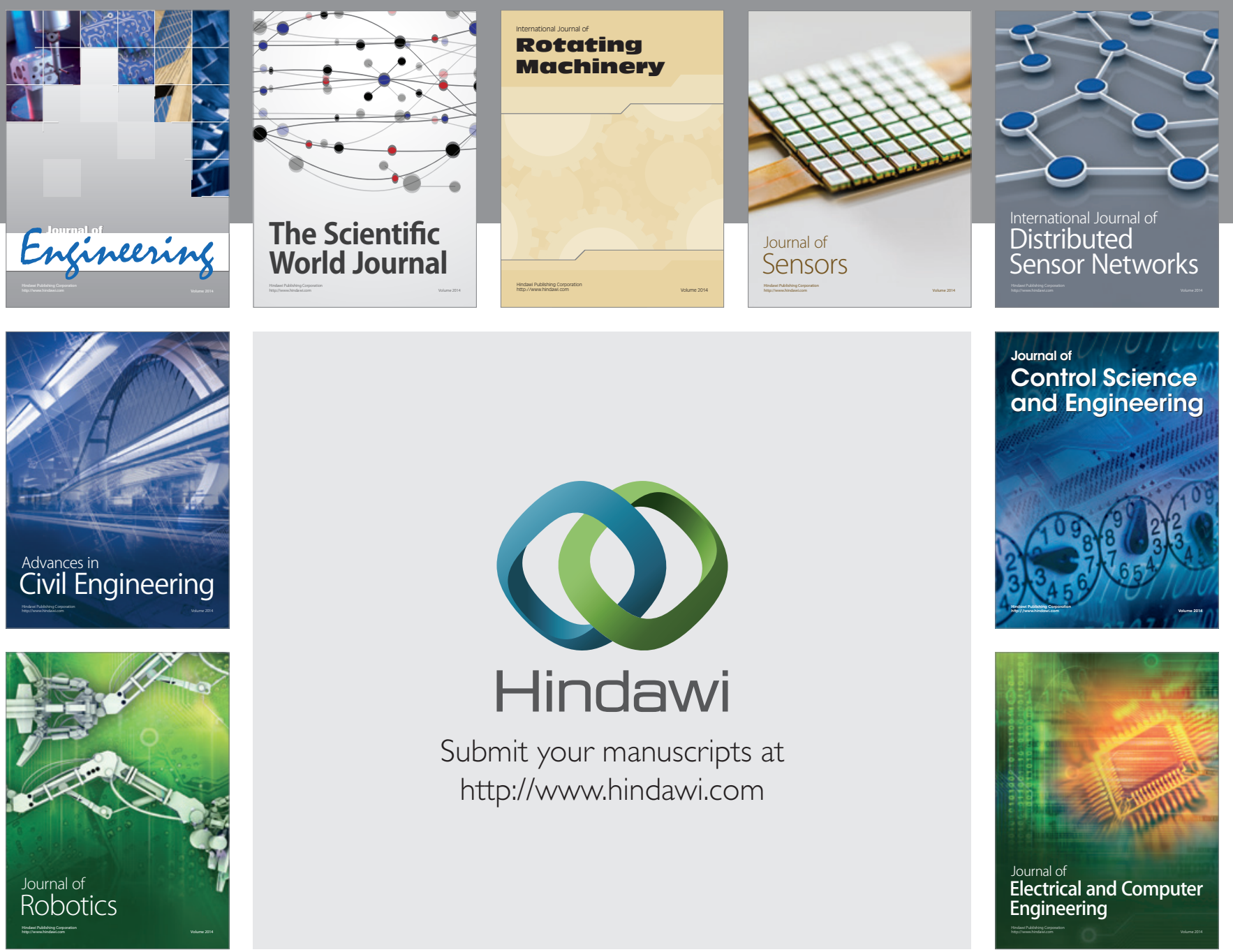

Submit your manuscripts at

http://www.hindawi.com
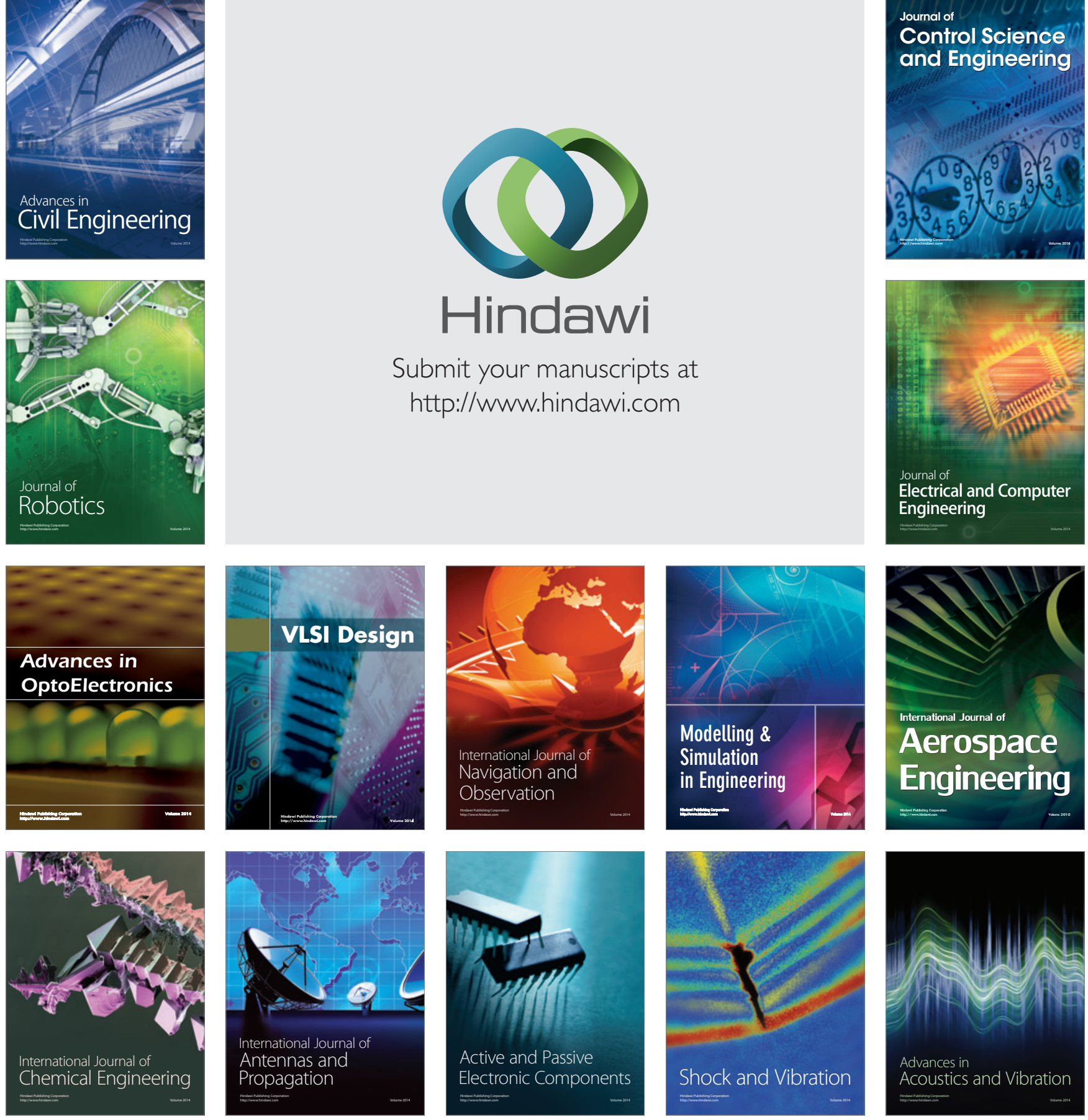